\title{
Bank-Laine Functions with Real Zeros
}

\author{
J. K. Langley ${ }^{1}$
}

Received: 28 February 2020 / Revised: 18 June 2020 / Accepted: 30 June 2020 /

Published online: 24 September 2020

(c) The Author(s) 2020

\section{Abstract}

Suppose that $E$ is a real entire function of finite order with zeros which are all real but neither bounded above nor bounded below, such that $E^{\prime}(z)= \pm 1$ whenever $E(z)=0$. Then either $E$ has an explicit representation in terms of trigonometric functions or the zeros of $E$ have exponent of convergence at least 3. An example constructed via quasiconformal surgery demonstrates the sharpness of this result.

Keywords Bank-Laine function · Entire function $\cdot$ Zeros

Mathematics Subject Classification 30D20 · 30D35

\section{Introduction}

For a non-constant entire function $f$, denote by

$$
\rho(f)=\limsup _{r \rightarrow+\infty} \frac{\log ^{+} T(r, f)}{\log r}, \quad \lambda(f)=\limsup _{r \rightarrow+\infty} \frac{\log ^{+} N\left(r, \frac{1}{f}\right)}{\log r} \leq \rho(f),
$$

its order of growth and the exponent of convergence of its zeros [10]. In their seminal paper [1], Bank and Laine proved several landmark results on the oscillation of solutions of

$$
y^{\prime \prime}+A(z) y=0,
$$

in which $A$ is an entire function. Their approach was based on taking linearly independent solutions $f_{1}, f_{2}$ of (1), normalised so as to have Wronskian $W\left(f_{1}, f_{2}\right)=$

Dedicated to the memory of Stephan Ruscheweyh.

Communicated by Vladimir V. Andrievskii.

$凶 \quad$ J. K. Langley

james.langley@nottingham.ac.uk

1 School of Mathematical Sciences, University of Nottingham, Nottingham, England NG7 2RD, UK 
$f_{1} f_{2}^{\prime}-f_{1}^{\prime} f_{2}=1$, and then considering the product $E=f_{1} f_{2}$, which satisfies

$$
4 A=\left(\frac{E^{\prime}}{E}\right)^{2}-2 \frac{E^{\prime \prime}}{E}-\frac{1}{E^{2}} .
$$

In particular, it was shown in [1] that if $\lambda(E)+\rho(A)<+\infty$ then $\rho(E)<+\infty$, whereas if $A$ is transcendental then the quotient $U=f_{1} / f_{2}$ always has infinite order, since [14, Ch. 6]

$$
S_{U}(z)=\frac{U^{\prime \prime \prime}(z)}{U^{\prime}(z)}-\frac{3}{2}\left(\frac{U^{\prime \prime}(z)}{U^{\prime}(z)}\right)^{2}=2 A,
$$

where $S_{U}(z)$ is the Schwarzian derivative. The following results were proved by Bank and Laine, Rossi and Shen [1,22,23].

Theorem 1.1 ([1,22,23]) Let A be an entire function, let $f_{1}, f_{2}$ be linearly independent solutions of (1) and let $E=f_{1} f_{2}$, so that $\lambda(E)=\max \left\{\lambda\left(f_{1}\right), \lambda\left(f_{2}\right)\right\}$.

(i) If $A$ is a polynomial of degree $n>0$ then $\lambda(E)=(n+2) / 2$.

(ii) If $\lambda(E)<\rho(A)<+\infty$ then $\rho(A) \in \mathbb{N}=\{1,2, \ldots\}$.

(iii) If $A$ is transcendental and $\rho(A) \leq 1 / 2$ then $\lambda(E)=+\infty$, while if $1 / 2<\rho(A)<$ 1 then

$$
\frac{1}{\rho(A)}+\frac{1}{\lambda(E)} \leq 2 .
$$

Theorem 1.1(ii) inspired the Bank-Laine conjecture, to the effect that if $A$ is a transcendental entire function and $f_{1}, f_{2}$ are linearly independent solutions of (1) with $\lambda\left(f_{1} f_{2}\right)$ finite then $\rho(A) \in \mathbb{N} \cup\{+\infty\}$. This conjecture has recently been disproved, however, in the first of two remarkable papers of Bergweiler and Eremenko [4,5] which use quasiconformal constructions; in the second of these they show that equality is possible in (4), for every choice of $\rho(A) \in(1 / 2,1)$.

The main thrust of this paper concerns the location of zeros of Bank-Laine functions, these being entire functions $E$ such that $E(z)=0$ implies $E^{\prime}(z)= \pm 1$. By [2, Lem. C], an entire function $E$ is a Bank-Laine function if and only if $E=f_{1} f_{2}$, where $f_{1}, f_{2}$ are linearly independent and solve (1), with $W\left(f_{1}, f_{2}\right)=1$ and $A$ entire, satisfying (2). Although a Bank-Laine function with unrestricted growth may have arbitrary zeros, subject only to these having no finite limit point [24], the following is a combination of results from $[6,18]$.

Theorem 1.2 ([6,18]) Let $E$ be a Bank-Laine function of finite order, with infinitely many zeros, all real, and denote by $n(r)$ the number of zeros of $E$ in $[-r, r]$. Then $n(r) \neq o(r)$ as $r \rightarrow+\infty$. If, in addition, all zeros of $E$ are positive, then $\lambda(E) \geq 3 / 2$.

The first assertion of Theorem 1.2 is evidently sharp, because of $\sin z$, and so is the second, a suitable example having been constructed in [18] using quasiconformal methods. The next theorem will considerably strengthen Theorem 1.2 in the case where $E$ is a real Bank-Laine function of finite order with real zeros, these neither bounded above nor bounded below. 
Theorem 1.3 Let E be a real Bank-Laine function of finite order, with only real zeros, these neither bounded above nor bounded below, and let $A$ be the associated coefficient function in (1) and (2). Then one of the following holds:

(i) there exist $\eta, \omega_{1}, \omega_{2} \in \mathbb{R}$ such that $\eta \sin \left(\omega_{1}-\omega_{2}\right) \neq 0$ and

$$
A=\eta^{2}, \quad E(z)= \pm \frac{\sin \left(\eta z-\omega_{1}\right) \sin \left(\eta z-\omega_{2}\right)}{\eta \sin \left(\omega_{1}-\omega_{2}\right)} ;
$$

(ii) $A$ is transcendental and $\lambda(E) \geq 3$, with $\rho(E)=\rho(A)=3$ if $\lambda(E)=3$.

Note that Hellerstein, Shen and Williamson [12] proved that if $A$ is a non-constant polynomial then (1) cannot have linearly independent solutions with only real zeros (see also $[9,26,27])$. A simple example illustrating (i) is given by

$$
A=\frac{1}{4}, \quad E(z)=\sin z=2 \sin \left(\frac{z}{2}\right) \cos \left(\frac{z}{2}\right)=-2 \sin \left(\frac{z}{2}\right) \sin \left(\frac{z}{2}-\frac{\pi}{2}\right)
$$

while $E(z)=\pi^{-1} e^{2 \pi i z^{2}} \sin \pi z$ shows that the hypothesis that $E$ is real entire is not redundant in Theorem 1.3. The sharpness of the result will be demonstrated in Sect. 4 , in which the quasiconformal techniques of [18] will be adapted to construct a real Bank-Laine function $E$, whose zeros are real but neither bounded above nor bounded below, such that $E$ and the function $A$ in (1) and (2) satisfy $\lambda(E)=\rho(E)=\rho(A)=3$.

\section{Preliminaries}

Let $G$ be a transcendental meromorphic function in the plane and suppose that $G(z) \rightarrow a \in \mathbb{C} \cup\{\infty\}$ as $z \rightarrow \infty$ along a path $\gamma$; then the inverse $G^{-1}$ is said to have a transcendental singularity over the asymptotic value $a[3,21]$. If $a \in \mathbb{C}$ then for each $\varepsilon>0$ there exists a component $\Omega=\Omega(a, \varepsilon, G)$ of the set $\{z \in \mathbb{C}:|G(z)-a|<\varepsilon\}$ such that $\gamma \backslash \Omega$ is bounded, and each such $\Omega$ is called a neighbourhood of the singularity [3]. Two paths $\gamma, \gamma^{\prime}$ on which $G(z) \rightarrow a$ determine distinct singularities if the corresponding components $\Omega(a, \varepsilon, G), \Omega^{\prime}(a, \varepsilon, G)$ are disjoint for some $\varepsilon>0$. The singularity is called direct [3] if $\Omega(a, \varepsilon, G)$, for some $\varepsilon>0$, contains no zeros of $G-a$, and $\operatorname{logarithmic}$ if there exists $\varepsilon>0$ such that $\log 1 /(G-a)$ maps $\Omega(a, \varepsilon, G)$ conformally onto the half-plane $\operatorname{Re} w>\log 1 / \varepsilon$. Transcendental singularities over $\infty$ may be classified using $1 / G$.

Denote by $B(a, r)$ the open disc of centre $a \in \mathbb{C}$ and radius $r$, and by $C l(D)$ the closure, with respect to the finite plane, of $D \subseteq \mathbb{C}$.

Proposition 2.1 Let $f_{1}, f_{2}$ be linearly independent solutions of (1), in which $A$ is a transcendental entire function of finite order, and assume that $W\left(f_{1}, f_{2}\right)=1$ and the zeros of $E=f_{1} f_{2}$ have finite exponent of convergence. Write

$$
U=\frac{f_{2}}{f_{1}}, \quad \frac{U^{\prime}}{U}=\frac{W\left(f_{1}, f_{2}\right)}{f_{1} f_{2}}=\frac{1}{E}, \quad F(z)=\frac{E(z)}{z} .
$$


Then the following statements hold.

(A) $U^{-1}$ has finitely many transcendental singularities over finite non-zero values.

(B) $U$ has no critical values and finitely many asymptotic values.

(C) Let $\Omega$ be a neighbourhood of a transcendental singularity of $U^{-1}$ over $\alpha \in \mathbb{C} \backslash\{0\}$. Then $\Omega$ contains a neighbourhood of a direct transcendental singularity of $F^{-1}$ over $\infty$, as well as a path tending to infinity on which $U(z) \rightarrow \alpha$ and $F(z) \rightarrow \infty$.

(D) $U^{-1}$ has infinitely many logarithmic singularities over 0 or $\infty$.

(E) Let $\gamma$ be a path tending to infinity on which $U(z)$ tends to 0 or $\infty$. Then $F(z)$ tends to 0 on $\gamma$.

Proof The fact that $U$ has no critical values is well known, and holds since $U^{\prime} / U=$ $1 / E \neq 0$ and all zeros and poles of $U$ are simple. Thus (B) follows from (A), and (A) from [17, Thm. 1.3], because the Bank-Laine equation (2) implies that $E$ has finite order [1].

To prove (C) requires an argument from [18, Lem. 5.2]. Since the singular value $\alpha$ of $U^{-1}$ is isolated, the singularity must be logarithmic [21, p. 287]. Hence there exist $M>0$ and a component $\Omega_{M} \subseteq \Omega$ of $\{z \in \mathbb{C}:|U(z)-\alpha|<1 / M\}$ which is mapped univalently by $v=\log 1 /(U(z)-\alpha)$ onto the half-plane $H_{0}$ given by $\operatorname{Re} v>\log M$. It may be assumed that $M$ is so large that $\Omega_{M} \cap B(0,1)=\emptyset$. Let $\phi: H_{0} \rightarrow \Omega_{M}$ be the inverse function and for $v \in H_{0}$ write

$$
U(z)=\alpha+e^{-v}, \quad E(z)=\frac{U(z)}{U^{\prime}(z)}=\frac{\alpha+e^{-v}}{-e^{-v}} \cdot \phi^{\prime}(v)=-\left(1+\alpha e^{v}\right) \phi^{\prime}(v) .
$$

Bieberbach's theorem and Koebe's one-quarter theorem [11, Ch. 1] give

$$
\left|\frac{\phi^{\prime \prime}(v)}{\phi^{\prime}(v)}\right| \leq \frac{4}{\operatorname{Re} v-\log M} \text { and } \quad\left|\frac{\phi^{\prime}(v)}{\phi(v)}\right| \leq \frac{4 \pi}{\operatorname{Re} v-\log M}
$$

on $H_{0}$, and so there exists $c_{1}>0$ such that, as $v \rightarrow+\infty$ on $\mathbb{R}$, by (7),

$$
|z|=|\phi(v)|=o\left(v^{c_{1}}\right)=o\left(e^{v}\left|\phi^{\prime}(v)\right|\right)=o(|E(z)|), \quad \frac{z}{E(z)}=\frac{1}{F(z)}=o(1) .
$$

Thus $U(z) \rightarrow \alpha$ and $F(z) \rightarrow \infty$ as $z \rightarrow \infty$ on the image under $\phi$ of the interval $[2+\log M,+\infty)$. On the other hand, if $\operatorname{Re} v=1+\log M$ then, again by (7),

$$
|F(z)| \leq(1+|\alpha| M e)\left|\frac{\phi^{\prime}(v)}{\phi(v)}\right| \leq(1+|\alpha| M e) 4 \pi .
$$

Hence there exist large positive $M_{0}, M_{1}$ and a component $C_{0}$ of $\left\{v \in H_{0}\right.$ : $\left.|F(\phi(v))|>M_{0}\right\}$ containing an interval $\left[M_{1},+\infty\right)$, such that $C l\left(C_{0}\right) \subseteq H_{0}$, and $\phi\left(C_{0}\right)$ is the required neighbourhood of a direct singularity of $F^{-1}$ over $\infty$.

Next, (D) follows from (A) and the result of Nevanlinna-Elfving [7,20], which implies that if $U^{-1}$ has finitely many transcendental singularities then its Schwarzian derivative $2 A$ must be a rational function, contrary to hypothesis (see also [16]). 
Finally, to prove (E), take a path $\gamma$ tending to infinity on which $V(z) \rightarrow \infty$, where $V$ is $U$ or $1 / U$. Since $\infty$ is not a limit point of singular values of $V^{-1}$, a standard estimate (see [8] or [25, Sect. 6]) gives positive constants $c_{2}, c_{3}$ such that, as $z$ tends to infinity on $\gamma$,

$$
\frac{1}{|F(z)|}=\left|\frac{z}{E(z)}\right|=\left|\frac{z V^{\prime}(z)}{V(z)}\right| \geq c_{2} \log \left|\frac{V(z)}{c_{3}}\right| \rightarrow+\infty .
$$

\section{Proof of Theorem 1.3}

Suppose that $E$ and $A$ are as in the hypotheses. Then there exist solutions $f_{1}, f_{2}$ of (1) such that $W\left(f_{1}, f_{2}\right)=1$ and $E=f_{1} f_{2}$. Furthermore, $A \neq \equiv 0$ and each $f_{j}$ has infinitely many zeros on each of the positive and negative real axes, since $f_{j}(z)=0$ gives $E^{\prime}(z)=(-1)^{j}$ and the sign of $E^{\prime}$ at successive zeros must alternate.

If $A=\eta^{2}$ is constant, then each $f_{j}$ is a linear combination of $e^{i \eta z}$ and $e^{-i \eta z}$; moreover, since the $f_{j}$ have infinitely many real zeros, $\eta$ must be real, and

$f_{j}(z)=A_{j} \sin \left(\eta z-\omega_{j}\right)$, with $A_{j}, \omega_{j}$ constants and $\omega_{j}$ real, which gives $E\left(\omega_{1} / \eta\right)=0$ and forces $E^{\prime}\left(\omega_{1} / \eta\right)= \pm 1$ and (5). Assume henceforth that $A$ is non-constant.

\section{Lemma 3.1 The function A is transcendental.}

Proof This follows from results in [9,12,26,27], and may be proved via the following slight modification of $[18$, Lem. 5.1]. Suppose that $A$ is a polynomial in (1), nonconstant by assumption, satisfying $A(z)=a_{n} z^{n}(1+o(1))$ as $z \rightarrow \infty$. Then there are $n+2>2$ critical rays given by $\arg z=\theta^{*}$, where $a_{n} e^{i(n+2) \theta^{*}}$ is real and positive, and a combination of the Liouville transformation

$$
Y(Z)=A(z)^{1 / 4} y(z), \quad Z=\int^{z} A(t)^{1 / 2} d t,
$$

with Hille's asymptotic method [13] generates linearly independent principal solutions of (1) given by $A(z)^{-1 / 4} e^{ \pm i Z}(1+o(1))$ on sectors symmetric about these rays. On one side of the critical ray, one of these principal solutions is large, while the other is small, these roles being reversed as the ray is crossed. Since the $f_{j}$ have infinitely many positive zeros, the positive real axis must be one of these $n+2$ critical rays, and each $f_{j}$ must be a non-trivial linear combination of the two principal solutions and thus large in both adjacent sectors. Let $L$ be the first other critical ray encountered on moving counter-clockwise from the positive real axis. Then $L$ is not the negative real axis, as $n>0$. Since the $f_{j}$ have only real zeros, both must change from large to small as $L$ is crossed. But $f_{1}, f_{2}$ cannot be small in the same sector, because $W\left(f_{1}, f_{2}\right)=1$.

Assume henceforth that $A$ is transcendental, but that $\lambda(E)=\lambda \leq 3$. Then the canonical product $\Pi_{0}$ over the zeros of $E$ has order $\lambda$, and there exists a real polynomial 
$P_{0}$ with $E=\Pi_{0} \exp \left(P_{0}\right)$. If $P_{0}$ has degree greater than $\lambda$, then the zeros of $E$ have Nevanlinna deficiency $\delta(0, E)=1$ [10, p. 42], contradicting [6, Thm. 4.1] (see also [15, Thm. 2.1]). Assume henceforth that $E$ has order $\rho(E)=\lambda \leq 3$ : then $\rho(A) \leq$ $\lambda \leq 3$ by (2).

Define $U$ and $F$ by (6). Since $U^{\prime} / U=1 / E$ is real, there exists $\theta \in \mathbb{R}$ such that $U=$ $f_{2} / f_{1}=e^{2 i \theta} U_{0}$, with $U_{0}$ real meromorphic. Replacing $f_{1}$ by $f_{1} e^{i \theta}$ and $f_{2}$ by $f_{2} e^{-i \theta}$ leaves $E$ unchanged; hence it may be assumed that $\theta=0$ and $U$ is real meromorphic. By Proposition $2.1, U^{-1}$ has finitely many transcendental singularities over finite non-zero values, but infinitely many transcendental singularities over 0 or $\infty$.

\section{Lemma 3.2 $U^{-1}$ has at least four logarithmic singularities over finite non-real values.}

Proof Note that it is not asserted that the corresponding four asymptotic values must all be distinct. Take zeros $x_{0}, x_{1}, x_{2} \in \mathbb{R}$ of $f_{2}$, with $0<x_{0}<x_{1}<x_{2}$, and the supremum $R$ of all $r>0$ such that the branch of $U^{-1}$ mapping 0 to $x_{1}$ extends analytically to $B(0, r)$. Then $R<+\infty$ and $U$ maps a simply connected domain $\Omega_{1}$, with $x_{1} \in \Omega_{1}$, univalently onto $B(0, R)$. Moreover, $U^{-1}$ has a singularity over some $\alpha$ with $|\alpha|=R$, and so $\Omega_{1}$ contains a path $\gamma$ which tends to infinity, mapped by $U$ onto the half-open line segment $[0, \alpha)$. If $\alpha \in \mathbb{R}$ then, since $U$ is real meromorphic and univalent on $\Omega_{1}$, the path $\gamma$ must be $\left(-\infty, x_{1}\right]$ or $\left[x_{1},+\infty\right)$, a contradiction since $x_{0}, x_{2} \notin \gamma$. Hence $\alpha \notin \mathbb{R}$ and so $U^{-1}$ has logarithmic singularities over $\alpha$ and $\bar{\alpha}$, by Proposition 2.1(B) and [21, p. 287].

Suppose now that $U^{-1}$ has no other logarithmic singularities over finite non-real values. Then, without loss of generality, there exist neighbourhoods $\Omega_{\alpha} \subseteq H^{+}$and $\Omega_{\bar{\alpha}} \subseteq H^{-}$of the singularities over $\alpha$ and $\bar{\alpha}$ respectively, where $H^{+}, H^{-}$denote the upper and lower half-planes. The argument of the previous paragraph shows that all but finitely many zeros of $f_{2}$ are joined to $\Omega_{\alpha}$ by a path which is mapped by $U$ onto $[0, \alpha)$, and to $\Omega_{\bar{\alpha}}$ by a path mapped onto $[0, \bar{\alpha})$. Since the set of zeros of $f_{2}$ is neither bounded above nor bounded below, this excludes transcendental singularities of $U^{-1}$ over $\infty$, and an almost identical argument applied to $f_{1}$ rules out transcendental singularities of $U^{-1}$ over 0 . This contradiction proves the lemma.

Thus, without loss of generality, there exist disjoint neighbourhoods $U_{1}, U_{2} \subseteq H^{+}$ and $U_{3}, U_{4} \subseteq H^{-}$of singularities of $U^{-1}$ over values $\alpha_{j} \in \mathbb{C} \backslash \mathbb{R}, j=1, \ldots, 4$. Proposition 2.1 delivers for each $j$ a path $\tau_{j} \subseteq U_{j}$ on which $U(z) \rightarrow \alpha_{j}$ and $F(z) \rightarrow$ $\infty$. Take a circle $|z|=R$, with $R$ large, which meets all four $\tau_{j}$ and on which $F$ has no zeros. Thus $|F(z)|$ is bounded below on the union of the circle and the $\tau_{j}$. Since $\tau_{1}, \tau_{2}$ lie in neighbourhoods of distinct singularities, while $U$ has only real poles, there must exist a path tending to infinity in $|z|>R$, lying between $\tau_{1}$ and $\tau_{2}$, on which $U(z) \rightarrow \infty$. Because $F$ has only real zeros, Proposition 2.1 now gives neighbourhoods $V_{j} \subseteq U_{j}$, for $j=1, \ldots, 4$, of direct singularities of $F^{-1}$ over $\infty$, and neighbourhoods $V_{5} \subseteq H^{+}$and $V_{6} \subseteq H^{-}$of direct singularities of $F^{-1}$ over 0 .

This gives positive constants $M_{j}$ and non-constant, non-negative subharmonic functions $u_{1}, \ldots, u_{6}$, with pairwise disjoint supports $V_{j}$, such that $u_{j}=\log \left|F / M_{j}\right|$ on $V_{j}$, for $j=1, \ldots, 4$, while $u_{j}=\log \left|M_{j} / F\right|$ on $V_{j}$, for $j=5,6$. Thus $u_{1}, \ldots, u_{4}$ have order $\rho\left(u_{j}\right) \leq \rho(F) \leq \rho(E)=\lambda(E) \leq 3$. Moreover, $u_{5}, u_{6}$ have order 
$\rho\left(u_{j}\right) \leq \rho(A) \leq 3$, because (2) and Poisson's formula yield as $r \rightarrow+\infty$, for $k=5,6$,

$$
\begin{aligned}
\max \left\{u_{k}(z):|z|=r\right\} & \leq \frac{3}{2 \pi} \int_{0}^{2 \pi} u_{k}\left(2 r e^{i t}\right) d t \leq 3 m\left(2 r, \frac{1}{F}\right)+O(1) \\
& \leq 3 m\left(2 r, \frac{1}{E}\right)+O(\log r) \leq 3 T(2 r, A)+O(\log r) .
\end{aligned}
$$

For $j=1, \ldots, 6$ and $t>0$ let $\theta_{j}(t)$ be the angular measure of $\{z \in \mathbb{C}:|z|=$ $\left.t, u_{j}(t)>0\right\}$. Let $S$ be large and positive: then a well known consequence of Carleman's estimate for harmonic measure [27, pp. 116-117] gives, as $r \rightarrow+\infty$,

$$
\begin{aligned}
36 \log \frac{r}{S} & =\int_{S}^{r}\left(\sum_{j=1}^{6} 1\right)^{2} \frac{d t}{t} \leq \int_{S}^{r}\left(\sum_{j=1}^{6} \theta_{j}(t)\right)\left(\sum_{j=1}^{6} \frac{1}{\theta_{j}(t)}\right) \frac{d t}{t} \\
& \leq 2 \sum_{j=1}^{6} \int_{S}^{r} \frac{\pi}{t \theta_{j}(t)} d t \leq 2 \sum_{j=1}^{6} \log \left(\max \left\{u_{j}(z):|z|=2 r\right\}\right)+O(1) \\
& \leq 2 \sum_{j=1}^{6}\left(\rho\left(u_{j}\right)+o(1)\right) \log r \leq(8 \lambda(E)+4 \rho(A)+o(1)) \log r \\
& \leq(36+o(1)) \log r .
\end{aligned}
$$

It follows at once that $\lambda(E)=\rho(E)=\rho(A)=3$.

\section{A Real Bank-Laine Function with Real Zeros}

The construction of an example demonstrating that Theorem 1.3 is sharp starts with the following.

\section{Lemma 4.1 The Möbius transformation}

$$
w=T(v)=\frac{e^{i \pi / 4}\left(1+e^{i \pi / 4} v\right)}{1-e^{-i \pi / 4} v}=\frac{e^{-i \pi / 4}\left(v-e^{i 3 \pi / 4}\right)}{v-e^{i \pi / 4}}
$$

satisfies

$$
T\left(e^{i \pi / 4}\right)=\infty, \quad T\left(e^{i 3 \pi / 4}\right)=0, \quad T(i)=-1, \quad T(0)=e^{i \pi / 4}, \quad T(\infty)=e^{-i \pi / 4} .
$$

In addition, $T$ maps the unit circle $|v|=1$ onto $\mathbb{R} \cup\{\infty\}$, with $\operatorname{Im} T(v)>0$ for $|v|<1$. Moreover, $\operatorname{Re} v=0$ implies that $|T(v)|=1$, while $|T(v)|>1$ for $\operatorname{Re} v>0$, and $T$ maps the line segment $[0, i]$ onto the counter-clockwise circular arc from $e^{i \pi / 4}$ to -1 . 
Proof All assertions follow from (9) and the following observations: first, (8) implies that $|T(v)|>1$ precisely when $v$ is further from $e^{i 3 \pi / 4}$ than from $e^{i \pi / 4}$; second, as $v$ describes the positive imaginary axis, $w$ travels around the unit circle from $e^{i \pi / 4}$ to $e^{-i \pi / 4}$ via -1 .

Lemma 4.2 Write $u=s+i t$ with $s, t \in \mathbb{R}$. Then the locally univalent functions

$$
f_{1}(u)=e^{i \pi / 4} \exp (\sqrt{2} v), \quad f_{2}(u)=T(v), \quad v=e^{i u}
$$

have the following properties:

(A) $\operatorname{Im} f_{2}(u)>0$ for $t>0$, and $f_{2}$ is a piecewise increasing mapping from $\mathbb{R}$ to $\mathbb{R} \cup\{\infty\}$

(B) $f_{2}$ has asymptotic values $e^{ \pm i \pi / 4}$ and poles at $u=(2 k+1 / 4) \pi, k \in \mathbb{Z}$, as well as zeros at $u=(2 k+3 / 4) \pi, k \in \mathbb{Z}$;

(C) for $j=1,2$, the function $\log \left|f_{j}(u)\right|$ is positive for $-\pi / 2<s<\pi / 2$, and negative if $-3 \pi / 2<s<-\pi / 2$ or $\pi / 2<s<3 \pi / 2$;

(D) $f_{1}$ maps the vertical line $\gamma_{1}$ given by $s=-\pi$ onto the open line segment $\left(0, e^{i \pi / 4}\right)$ and there exists a path $\gamma_{2}$, starting at $3 \pi / 4$ and tending to infinity in the half-strip $\pi / 2<s<3 \pi / 2,0 \leq t<+\infty$, which is mapped by $f_{2}$ onto the half-open line segment $\left[0, e^{i \pi / 4}\right)$.

Proof (A) and (B) follow from Lemma 4.1, as does (C) for $f_{2}$, while (C) for $f_{1}$ is an immediate consequence of the formula $\log \left|f_{1}(u)\right|=\sqrt{2} e^{-t} \cos s$. The assertion (D) for $f_{1}$ is obvious, while (D) for $f_{2}$ follows from (A) and analytic continuation of $f_{2}^{-1}$, the only singular values of which are the two values omitted by $f_{2}$, namely $e^{ \pm i \pi / 4}$.

The construction will proceed by first forming, on the sector $0<\arg u<3 \pi / 2$, a quasiregular mapping which is $f_{1}(u)$ for $\operatorname{Re} u \leq-\pi / 2$ and $f_{2}(u)$ for $\operatorname{Re} u \geq \pi / 2$. A modification of this mapping will be pulled back to the first quadrant, which will then permit extension via double reflection to a quasimeromorphic mapping on the whole plane. Application of the Teichmüller-Belinskii theorem [19] will result in a locally univalent meromorphic function $U$ for which $E=U / U^{\prime}$ will be the required Bank-Laine function. To this end, set

$$
\begin{aligned}
& D_{0}=\left\{u \in \mathbb{C}: 0<|u|<+\infty, 0<\arg u<\frac{3 \pi}{2}\right\}, \\
& D_{1}=E_{1} \cup E_{2}, \\
& E_{1}=\left\{s+i t:-\frac{\pi}{2}<s<0,-\infty<t<+\infty\right\}, \\
& E_{2}=\left\{s+i t:-\frac{\pi}{2}<s<\frac{\pi}{2}, 0<t<+\infty\right\}, \\
& D_{2}=\left\{v \in \mathbb{C}: 0<|v|<+\infty,-\frac{\pi}{2}<\arg v<0\right\}, \\
& D_{3}=D_{2} \cup\{\zeta \in \mathbb{C}:|\zeta|<1, \operatorname{Re} \zeta>0\}, \\
& D_{4}=\{\sigma+i \tau: 0<\sigma<+\infty,-\infty<\tau<\pi\} .
\end{aligned}
$$

The following is [18, Lem. 6.1]. 
Lemma 4.3 ([18]) Let $h:(-\infty, 1] \rightarrow(-\infty, 0]$ be a continuous bijection, such that $h(1)=0$ while $h^{\prime}$ is continuous and has positive upper and lower bounds for $-\infty<y<1$ (that is, there exists $\varepsilon>0$ such that $\varepsilon<h^{\prime}(y)<1 / \varepsilon$ for $-\infty<y<1$ ). Then there exists a homeomorphism $\psi$ from the closure of $D_{3}$ to that of $D_{2}$, such that: (A) $\psi$ maps $D_{3}$ quasiconformally onto $D_{2}$, with $\psi(z) \rightarrow \infty$ and $\psi(z)=O(|z|)$ as $z \rightarrow \infty$ in $D_{3} ;(B) \psi(i y)=i h(y)$ for $-\infty<y \leq 1 ;(C) \psi(z)$ is real and strictly increasing as $z$ describes the boundary of $D_{3}$ clockwise from $i$ to infinity.

Lemma 4.4 With $D_{0}, D_{1}$ as in (11), and the $f_{j}$ as in Lemma 4.2, let $E_{0}=C l\left(D_{0}\right)$ and define $F$ on $E_{0} \backslash D_{1}$ by

$$
\begin{aligned}
& F(s+i t)=f_{1}(s+i t) \text { for }-\infty<s \leq-\frac{\pi}{2}, t \in \mathbb{R} \\
& F(s+i t)=f_{2}(s+i t) \text { for } \frac{\pi}{2} \leq s<+\infty, 0 \leq t<+\infty
\end{aligned}
$$

Then $F$ extends to a mapping from $E_{0}$ into the extended plane, continuous with respect to the spherical metric, with the following properties.

(i) $H=\log F$ maps $D_{1}$ quasiconformally onto $D_{4}$, with $H(\pi / 2)=i \pi$.

(ii) $F$ is locally injective on $E_{0}$.

(iii) Let $L_{0}$ be the path consisting of the line segment from $3 \pi / 4$ to 0 followed by the negative imaginary axis in the direction of $-i \infty$. Then $F(3 \pi / 4)=0$ and $F(u)$ is real and strictly decreasing as $u$ describes $L_{0}$, mapping $L_{0}$ onto the nonpositive real axis. Moreover, each $u_{0} \in L_{0}$ has $s_{0}>0$ such that $\operatorname{Im} F(u)>0$ on $D_{0} \cap B\left(u_{0}, s_{0}\right)$.

(iv) There exists $c>0$ such that $|F(u)| \leq \exp \exp (c|u|)$ for $u \in D_{0}$ lying on the circles $|u|=n \pi, n \in \mathbb{N}$.

Proof First, observe that $v=e^{i u}$ maps $D_{1}$ onto $D_{3}$, with $v(\pi / 2)=i$ and $v \rightarrow 0$ as $\operatorname{Im} u \rightarrow+\infty$, as well as $v \rightarrow \infty$ as $\operatorname{Im} u \rightarrow-\infty$. Indeed, the boundary of $D_{1}$ is mapped by $v=e^{i u}$ as follows: the line $\operatorname{Re} u=-\pi / 2$ to the negative imaginary axis; the half-line $\operatorname{Re} u=\pi / 2,0 \leq \operatorname{Im} u<+\infty$, to the segment $v=i y, 0<y \leq 1$; the interval $[0, \pi / 2] \subseteq \mathbb{R}$ to the arc of the unit circle from 1 to $i$; the negative imaginary axis to $(1,+\infty)$. Using Lemma 4.1 and the principal argument, set

$$
h(y)= \begin{cases}\frac{\pi}{4}+\sqrt{2} y & \text { for }-\infty<y \leq 0, \\ \arg T(i y)=\frac{\pi}{4}+\arg \left(\frac{1+e^{i \pi / 4} i y}{1-e^{-i \pi / 4} i y}\right)=\frac{\pi}{4}-i \log \left(\frac{1+e^{i 3 \pi / 4} y}{1+e^{-i 3 \pi / 4} y}\right) & \text { for } 0<y \leq 1 .\end{cases}
$$

Then $h(1)=\pi$ and, for $0<y<1$,

$$
h^{\prime}(y)=-i\left(\frac{e^{i 3 \pi / 4}}{1+e^{i 3 \pi / 4} y}-\frac{e^{-i 3 \pi / 4}}{1+e^{-i 3 \pi / 4} y}\right)=\frac{2 \sin \left(\frac{3 \pi}{4}\right)}{\left|1+e^{i 3 \pi / 4} y\right|^{2}}>0,
$$

so that $\lim _{y \rightarrow 1-} h^{\prime}(y)$ is finite but positive, and $\lim _{y \rightarrow 0+} h^{\prime}(y)=\sqrt{2}$, which leads to $h^{\prime}(0)=\sqrt{2}$. Thus $h$ is a continuous bijection from $(-\infty, 1]$ to $(-\infty, \pi]$ and $h^{\prime}$ exists 
and is continuous on $(-\infty, 1)$, with positive upper and lower bounds there. Applying Lemma 4.3 to $h(y)-\pi$ gives a homeomorphism $\psi$ from the closure of $D_{3}$ to that of the quadrant $D_{4}$ in (11), such that $\psi$ maps $D_{3}$ quasiconformally onto $D_{4}$, with $\psi(v)=O(|v|)$ as $v \rightarrow \infty$ in $D_{3}$ and $\psi(i y)=i h(y)$ for $-\infty<y \leq 1$. The function $G=\exp \circ \psi$ is then continuous on $C l\left(D_{3}\right)$ and satisfies, by (13),

$$
\begin{aligned}
G(v) & =\exp (i h(y))=e^{i \pi / 4} \exp (\sqrt{2} i y) \\
& =e^{i \pi / 4} \exp (\sqrt{2} v) \text { for } v=i y,-\infty<y \leq 0, \\
G(v) & =\exp (i h(y))=T(i y)=T(v) \text { for } v=i y, 0<y \leq 1 .
\end{aligned}
$$

Now set $F(u)=G\left(e^{i u}\right)$ on $C l\left(D_{1}\right)$. Then (8), (10), (12), (14) and the properties already noted of the mapping $v=e^{i u}$ from $D_{1}$ to $D_{3}$ ensure that $F$ is well-defined and continuous on $E_{0}$, and that (i) holds. Because $\psi$ is injective on $D_{3}$, and $|F|>1$ on $D_{1}$, Lemma 4.2(C) implies (ii). To establish (iii), observe first that $F(u)=f_{2}(u)$ is real and decreases from 0 to -1 as $u$ traverses the line segment from $3 \pi / 4$ to $\pi / 2$, by (9), (10) and Lemma 4.2(A). Next, as $u$ follows $L_{0}$ from $\pi / 2$ towards infinity, $v=e^{i u}$ describes the boundary of $D_{3}$ clockwise from $i$ to infinity, so that $\psi(v)$ describes the half-line $\{\sigma+i \pi: 0 \leq \sigma<+\infty\}$, by Lemma 4.3 applied to $h(y)-\pi$, and $F(u)=G(v)=\exp (\psi(v))$ travels from -1 along the negative real axis towards $-\infty$. Finally, to prove (iv), note first that (12) and Lemma 4.2 show that it is enough to bound $|F(u)|$ for $u \in D_{1}$, and hence it suffices to consider $G(v)$, which is continuous on $C l\left(D_{3}\right)$ and satisfies, as $v=e^{i u} \rightarrow \infty$ in $D_{3}$,

$$
|F(u)|=|G(v)| \leq \exp (|\psi(v)|) \leq \exp (O(|v|))=\exp \left(O\left(\left|e^{i u}\right|\right)\right) \leq \exp \exp (2|u|)
$$

Next, let $L$ be the quasiconformal mapping of the extended plane given by $L\left(r e^{i \theta}\right)=r e^{i g(\theta)}$ for $r>0$ and $0 \leq \theta \leq 2 \pi$, where $g$ is continuous, strictly increasing and piecewise linear with

$$
g(\theta)=\theta \quad \text { for } \quad 0 \leq \theta \leq \frac{\pi}{3}, \quad g(\pi)=\frac{\pi}{2}, \quad g(2 \pi)=2 \pi .
$$

Let $E_{3}$ be the component of $E_{0} \backslash\left(\gamma_{1} \cup \gamma_{2}\right)$ which contains $D_{1}$, where $\gamma_{1}, \gamma_{2}$ are as in Lemma 4.2(D), and set $V(u)=L(F(u))$ for $u \in E_{3}$, with $V(u)=F(u)$ on $E_{0} \backslash E_{3}$. Since $F$ maps $\gamma_{1} \cup \gamma_{2}$ into the segment $\left[0, e^{i \pi / 4}\right)$, on which $L$ is the identity, $V$ is well-defined and continuous on $E_{0}$, and quasiregular and non-zero on $D_{0}$, with $V(u)=F(u)=f_{2}(u) \in \mathbb{R} \cup\{\infty\}$ on $[3 \pi / 4,+\infty)$. Furthermore, $V$ maps the path $L_{0}$ in Lemma 4.4(iii) onto the non-negative imaginary axis, each $u_{0} \in L_{0}$ having $s_{0}>0$ such that $0<\arg V(u)<\pi / 2$ on $D_{0} \cap B\left(u_{0}, s_{0}\right)$.

Set $x_{0}=(3 \pi / 4)^{2 / 3} \in(0,+\infty)$, and on the quadrant $D_{5}$ given by $0<\arg z<\pi / 2$ write $\zeta=x_{0}+z^{2}$ and $u=\eta(z)=\zeta^{3 / 2}$, taking the principal branch. Then $\eta$ maps $D_{5}$ onto $D_{0}$ and extends continuously to $\partial D_{5}$, with 0 mapped to $3 \pi / 4$, the non-negative imaginary axis to the path $L_{0}$, and the non-negative real axis to $[3 \pi / 4,+\infty)$. Set $Y(z)=V(\eta(z))=V\left(\left(x_{0}+z^{2}\right)^{3 / 2}\right)$ on $D_{5}$ and extend $Y$ to $C l\left(D_{5}\right)$ by continuity. 
Then $Y$ maps the non-negative real axis into $\mathbb{R} \cup\{\infty\}$, with $Y(0)=V(3 \pi / 4)=0$, and is a bijection from the non-negative imaginary axis to itself. Double reflection, first across the imaginary axis and then across the real axis, extends $Y$ to the whole plane. The resulting function is locally injective in the plane, by Lemma 4.2(A) and the mapping properties of $V$ on $L_{0}$, and quasimeromorphic [19, Ch. I, Thm. 8.3]. Further, $Y$ now maps $\mathbb{R}$ into $\mathbb{R} \cup\{\infty\}$, and has only real zeros and poles. If $x$ is large and positive then $x$ is a zero or pole of $Y$ if and only if $\eta(x) \sim x^{3}$ is a zero or pole of $f_{2}$. Thus the set of zeros and poles of $Y$ is neither bounded above nor bounded below, and by Lemma 4.2 the number $n_{Y}(r)$ of these in $[-r, r]$ satisfies

$$
c_{1} r^{3} \leq n_{Y}(r) \leq c_{2} r^{3} \text { as } r \rightarrow+\infty
$$

in which the $c_{j}$ denote positive constants. Moreover, Lemma 4.4(iv) gives, for large $n \in \mathbb{N}$,

$$
\log ^{+} \log ^{+}|Y(z)|=O(n),
$$

initially for $z \in D_{5}$ with $\left|x_{0}+z^{2}\right|=(n \pi)^{2 / 3}$, and hence by reflection on a Jordan curve $\Gamma_{n}$ on which $|z| \sim(n \pi)^{1 / 3}$.

The remainder of the construction proceeds as in [4,18]. Let $E_{4}$ be the pre-image in $D_{5}$ of $D_{6}=\left\{u \in D_{0}:-2 \pi<\operatorname{Re} u<2 \pi\right\}$ under $u=\eta(z)$. If $E_{4}^{\prime}=\left\{z \in E_{4}\right.$ : $\left.|z|>R^{\prime}\right\}$, where $R^{\prime}$ is large, then writing $z=x+i y, u=\kappa+i \lambda$, with $x, y, \kappa, \lambda$ real, leads to

$$
\begin{aligned}
\int_{E_{4}^{\prime}} \frac{1}{|z|^{2}} d x d y & =\int_{\eta\left(E_{4}^{\prime}\right)} \frac{1}{\left|z \eta^{\prime}(z)\right|^{2}} d \kappa d \lambda=\int_{\eta\left(E_{4}^{\prime}\right)} \frac{1}{\left|9 z^{4}\left(x_{0}+z^{2}\right)\right|} d \kappa d \lambda \\
& \leq c_{3}+c_{4} \int_{u \in D_{6},|u|>1} \frac{1}{|u|^{2}} d \kappa d \lambda<+\infty
\end{aligned}
$$

Now let $F_{4}$ be the closure of the union of $E_{4}$ and its reflections across the real and imaginary axes. Then $Y$ is meromorphic off $F_{4}$ and (17) implies that the complex dilatation $\mu_{Y}$ of $Y$ satisfies

$$
\int_{1 \leq|z|<+\infty}\left|\frac{\mu_{Y}(z)}{z^{2}}\right| d x d y \leq \int_{1 \leq|z|<+\infty, z \in F_{4}} \frac{1}{|z|^{2}} d x d y<+\infty .
$$

Let $\phi$ be the unique quasiconformal homeomorphism of the extended plane which solves the Beltrami equation $\phi_{\bar{z}}=\mu_{Y} \phi_{z}$ a.e. and fixes each of 0,1 and $\infty$ [19]. In view of (18) and the Teichmüller-Belinskii theorem [19, Ch. V, Thm. 6.1], there exists $\alpha \in \mathbb{C} \backslash\{0\}$ with

$$
\phi(z) \sim \alpha z
$$

as $z \rightarrow \infty$. Furthermore, there exists a locally univalent meromorphic function $U$ such that $Y=U \circ \phi$ on $\mathbb{C}$, and writing $U_{1}(z)=\overline{U(\bar{z})}$ gives

$$
U(\phi(z))=Y(z)=\overline{Y(\bar{z})}=\overline{U(\phi(\bar{z}))}=U_{1}(\overline{\phi(\bar{z})})
$$


Thus $\phi(z)$ and $\overline{\phi(\bar{z})}$ have the same complex dilation a.e. and, since both fix 0,1 and $\infty$, they must agree, so that $\phi$ is real on $\mathbb{R}$ and $U$ is real meromorphic. Moreover, all zeros and poles of $U$ are real, and $E=U / U^{\prime}$ is a real Bank-Laine function with real zeros, these neither bounded above nor bounded below. Let $\Pi_{1}$ and $\Pi_{2}$ be the canonical products over the zeros and poles of $U$ respectively. Then (15) and (19) imply that $\Pi_{1}$ and $\Pi_{2}$ have order at most 3 , and that the associated coefficient function $A$ in (1) and (2) cannot be constant. There exists an entire function $Q$ such that

$$
U=\frac{\Pi_{1}}{\Pi_{2}} e^{Q}, \quad \frac{1}{E}=\frac{U^{\prime}}{U}=\frac{\Pi_{1}^{\prime}}{\Pi_{1}}-\frac{\Pi_{2}^{\prime}}{\Pi_{2}}+Q^{\prime} .
$$

By (16) and (19), the entire function $f_{0}=\Pi_{2} U$ satisfies, on the Jordan curve $\Gamma_{n}$,

$$
\left|f_{0}(\phi(z))\right|=\left|\Pi_{2}(\phi(z)) Y(z)\right| \leq C_{0} e^{|\phi(z)|^{4}} \exp \exp \left(C_{1} n\right) \leq \exp \exp \left(C_{2} n\right),
$$

in which the positive constants $C_{j}$ are independent of $n$, and so $\left|f_{0}(w)\right| \leq$ $\exp \exp \left(C_{2} n\right)$ for $w$ on $\phi\left(\Gamma_{n}\right)$. Since $\phi\left(\Gamma_{n}\right)$ encloses a circle $|w|=C_{3} n^{1 / 3}$, the maximum principle gives

$$
\log T\left(r, f_{0}\right) \leq \log \log M\left(r, f_{0}\right)=O\left(r^{3}\right) \text { as } r \rightarrow+\infty .
$$

On combination with (20) and the lemma of the logarithmic derivative [10], this leads to

$T\left(r, Q^{\prime}\right)=m\left(r, Q^{\prime}\right) \leq m\left(r, \frac{f_{0}^{\prime}}{f_{0}}\right)+m\left(r, \frac{\Pi_{1}^{\prime}}{\Pi_{1}}\right)+O(1)=O\left(r^{3}\right)$ as $r \rightarrow+\infty$.

Hence (20) implies that $\rho(E) \leq 3$, and $\lambda(E)=\rho(E)=\rho(A)=3$ by Theorem 1.3.

Acknowledgements The author thanks the referees for several helpful suggestions.

Open Access This article is licensed under a Creative Commons Attribution 4.0 International License, which permits use, sharing, adaptation, distribution and reproduction in any medium or format, as long as you give appropriate credit to the original author(s) and the source, provide a link to the Creative Commons licence, and indicate if changes were made. The images or other third party material in this article are included in the article's Creative Commons licence, unless indicated otherwise in a credit line to the material. If material is not included in the article's Creative Commons licence and your intended use is not permitted by statutory regulation or exceeds the permitted use, you will need to obtain permission directly from the copyright holder. To view a copy of this licence, visit http://creativecommons.org/licenses/by/4.0/.

\section{References}

1. Bank, S., Laine, I.: On the oscillation theory of $f^{\prime \prime}+A f=0$ where $A$ is entire. Trans. Amer. Math. Soc. 273, 351-363 (1982)

2. Bank, S., Laine, I.: On the zeros of meromorphic solutions of second-order linear differential equations. Comment. Math. Helv. 58, 656-677 (1983)

3. Bergweiler, W., Eremenko, A.: On the singularities of the inverse to a meromorphic function of finite order. Rev. Mat. Iberoamericana 11, 355-373 (1995) 
4. Bergweiler, W., Eremenko, A.: On the Bank-Laine conjecture. J. Eur. Math. Soc. 19, 1899-1909 (2017)

5. Bergweiler, W., Eremenko, A.: Quasiconformal surgery and linear differential equations. J. Analyse Math. 137, 751-812 (2019)

6. Drasin, D., Langley, J.K.: Bank-Laine functions via quasiconformal surgery, transcendental dynamics and complex analysis, In: London Math. Soc. Lecture Notes 348, Cambridge University Press, pp. 165-178 (2008)

7. Elfving, G.: Über eine Klasse von Riemannschen Flächen und ihre Uniformisierung. Acta Soc. Sci. Fenn. 2, 1-60 (1934)

8. Eremenko, A.E., Lyubich, MYu.: Dynamical properties of some classes of entire functions. Ann. Inst. Fourier Grenoble 42, 989-1020 (1992)

9. Gundersen, G.G.: On the real zeros of solutions of $f^{\prime \prime}+A(z) f=0$ where $f$ is entire. Ann. Acad. Sci. Fenn. Ser. A. I. Math. 11, 275-294 (1986)

10. Hayman, W.K.: Meromorphic Functions. Oxford at the Clarendon Press, Oxford (1964)

11. Hayman, W.K.: Multivalent Functions, 2nd Edition, Cambridge Tracts in Mathematics 110. Cambridge University Press, Cambridge (1994)

12. Hellerstein, S., Shen, L.-C., Williamson, J.: Real zeros of derivatives of meromorphic functions and solutions of second order differential equations. Trans. Amer. Math. Soc. 285, 759-776 (1984)

13. Hille, E.: Ordinary differential equations in the complex domain. Wiley, New York (1976)

14. Laine, I.: Nevanlinna theory and complex differential equations, de Gruyter Studies in Math. 15. Walter de Gruyter, Berlin/New York (1993)

15. Langley, J.K.: Bank-Laine functions with sparse zeros. Proc. Amer. Math. Soc. 129, 1969-1978 (2001)

16. Langley, J.K.: The Schwarzian derivative and the Wiman-Valiron property. J. Anal. Math. 130, 71-89 (2016)

17. Langley, J.K.: Transcendental singularities for a meromorphic function with logarithmic derivative of finite lower order. Comput. Methods Funct. Theory 19, 117-133 (2019)

18. Langley J.K: Bank-Laine functions, the Liouville transformation and the Eremenko-Lyubich class. J. Anal. Math (to appear)

19. Lehto, O., Virtanen, K.: Quasiconformal Mappings in the Plane, 2nd edn. Springer, Berlin (1973)

20. Nevanlinna, R.: Über Riemannsche Flächen mit endlich vielen Windungspunkten. Acta Math. 58, 295-373 (1932)

21. Nevanlinna, R.: Eindeutige Analytische Funktionen, 2nd edn. Springer, Berlin (1953)

22. Rossi, J.: Second order differential equations with transcendental coefficients. Proc. Amer. Math. Soc. 97, 61-66 (1986)

23. Shen, L.C.: Solution to a problem of S. Bank regarding the exponent of convergence of the solutions of a differential equation $f^{\prime \prime}+A f=0$. Kexue Tongbao 30, 1581-1585 (1985)

24. Shen, L.C.: Construction of a differential equation $y^{\prime \prime}+A y=0$ with solutions having prescribed zeros. Proc. Amer. Math. Soc. 95, 544-546 (1985)

25. Sixsmith, D.J.: Dynamics in the Eremenko-Lyubich class. Conform. Geom. Dyn. 22, 185-224 (2018)

26. Steinmetz, N.: Linear differential equations with exceptional fundamental sets II. Proc. Amer. Math. Soc. 117(2), 355-358 (1993)

27. Tsuji, M.: Potential Theory in Modern Function Theory. Maruzen, Tokyo (1959)

Publisher's Note Springer Nature remains neutral with regard to jurisdictional claims in published maps and institutional affiliations. 